\title{
Bild und Wort dans la conférence d'Aby Warburg sur les tapisseries Valois : méthode pour une Bildwissenschaft
}

\section{Katia Mazzucco}

Traducteur : Frédérique Borde et Renata Miotti

\section{OpenEdition}

\section{Journals}

Édition électronique

URL : http://journals.openedition.org/imagesrevues/2972

DOI : 10.4000/imagesrevues.2972

ISSN : 1778-3801

\section{Éditeur :}

Centre d'Histoire et Théorie des Arts, Groupe d'Anthropologie Historique de l'Occident Médiéval,

Laboratoire d'Anthropologie Sociale, UMR 8210 Anthropologie et Histoire des Mondes Antiques

Référence électronique

Katia Mazzucco, «Bild und Wort dans la conférence d'Aby Warburg sur les tapisseries Valois: méthode pour une Bildwissenschaft », Images Re-vues [En ligne], Hors-série 4 | 2013, document 14, mis en ligne le 30 janvier 2013, consulté le 01 février 2021. URL : http://journals.openedition.org/ imagesrevues/2972 ; DOI : https://doi.org/10.4000/imagesrevues.2972

Ce document a été généré automatiquement le 1 février 2021.

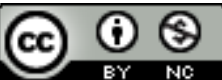

Images Re-vues est mise à disposition selon les termes de la Licence Creative Commons Attribution Pas d'Utilisation Commerciale 4.0 International. 


\section{Bild und Wort dans la conférence d'Aby Warburg sur les tapisseries Valois : méthode pour une Bildwissenschaft}

\section{Katia Mazzucco}

Traduction : Frédérique Borde et Renata Miotti

1 Le sujet de cet article est la Bilderreihe pour la conférence tenue par Aby Warburg à Florence en octobre 1927, une œuvre inédite qui problématise la définition d'une iconologie warburgienne ${ }^{1}$.

2 Lors de cette occasion scientifique,

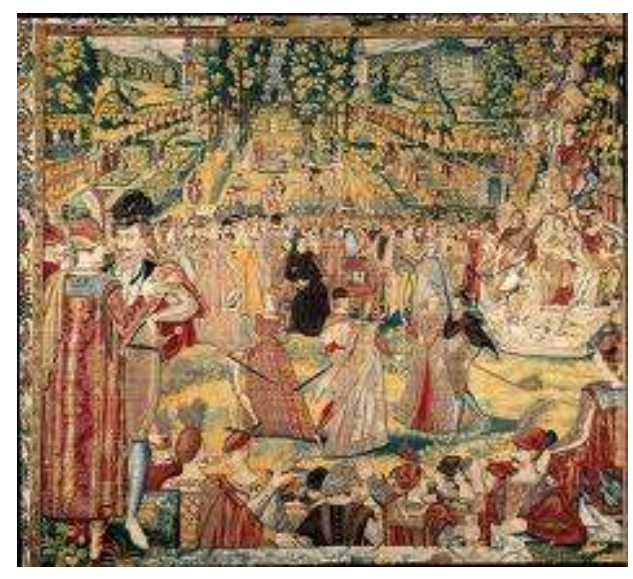

Warburg parle de l'objet de sa propre recherche, les fêtes médicéennes immortalisées sur les tapisseries des Valois à la Galerie des Offices et indique la nécessité d'une " étude iconologique " (ikonologischen Durcharbeitung)². La partie de la conférence consacrée au matériel documentaire visuel recueilli pour l'occasion débute avec le commentaire de ces images en tant «qu'instruments iconographiques» (ikonographische Hilfsmittel) ${ }^{3}$ pour la comparaison et l'identification des portraits.

3 J'introduis cette question centrale en mettant en évidence trois occurrences significatives du binôme expressif image/parole. Trois occurrences dans le travail 
d'Aby Warburg, pour mettre en évidence trois aspects sémantiques de ce binôme dans la pensée du chercheur.

\section{Einfühlenden Bildgedächtnisses}

Entre 1917 et 1918 Warburg expose à plusieurs occasions le résultat sans doute le plus complexe de sa recherche et, avec la publication en 1920, de l'essai Heidnisch-antike Weissagung in Wort und Bild zu Luthers Zeiten ${ }^{4}$, de sa prose.

5 L'auteur indique dans son essai une première contribution pour combler une lacune des études culturelles ou mieux pour indiquer un secteur de recherche restant à inventer, qui fasse dialoguer différentes perspectives disciplinaires - un perfectionnement de la méthode pour la Kulturwissenschaft qui utilise, comme ici, le dialogue entre science des religions et histoire de l'art ${ }^{5}$. Son analyse se penche, entre autres, en détail sur les libelles et les tracts illustrés de propagande de la Réforme. Considéré pour ses qualités de véhicule et de média, ce type de document reproductible fournit le terrain se prêtant à la présentation d'un binôme clé de la pensée et de la recherche warburgienne sur les mécanismes de la transmission et de la tradition culturelle, Wort und Bild (parole et image), justement. L'élément central de l'analyse, souligne l'auteur, réside dans le rôle tenu, à l'intérieur de ces dynamiques, par la «einfühlenden Bildgedächtnisses » (mémoire visuelle empathique) ${ }^{6}$.

Dans ses pages, complexes, consacrées à l'interprétation des images prophétiques et de l'utilisation politique du généthliaque luthérien, l'essai propose, entre autres, des éléments d'interprétation de celle qui peut être considérée l'icône de la science sans $n^{7}$ : il s'agit de la célèbre Melencolia d'Albrecht Dürer, cœur de l' étude Saturn and Melancholy de Klibansky, Panofsky, Saxl (1964) que Robert Klein allait qualifier d'illustration la plus efficace de la discipline créée par Warburg, «une discipline qui, contrairement à tant d'autres, existe, mais n'a pas de nom ${ }^{8}$.

\section{Dans la Bildersammlung}

7 «Wort und Bild» est la deuxième section du système de classification planifié autour de 1926-1927 pour la collection photographique Warburg de Hambourg9

La Photographic Collection du Warburg Institute de Londres constitue un outil unique pour la recherche iconographique in situ. Le système complexe de catégories de sujets est en effet l'un des éléments qui caractérise la collection. L'origine de la configuration actuelle, qui a été enrichie et subdivisée au fil des ans, proviendrait de la collaboration entre Edgar Wind et Rudolf Wittkower.

9 L'accès réduit aux sources directes de cette histoire, en d'autres termes la dissémination de documents en grande partie non identifiés, semble toutefois avoir mené à une prise en considération partielle de la configuration de la collection photographique jusqu'à l'intervention Wind-Wittkower et, suscité, de fait, le préjugé selon lequel il n'y avait pas de vrai système d'organisation pour la collection photographique de Hambourg. Des documents non identifiés jusqu'à présent, comprenant entre autre le plan original pour la photothèque d'Hambourg, donnent une autre version de l'histoire. 
10 Le cas de la collection photographique de la Bibliothèque Warburg de Hambourg connaît la même évolution que d'autres institutions pour l'histoire de l'art fondées à la fin du XIX ${ }^{e}$ siècle. Elle naît à la fin du siècle en tant que collection personnelle d'Aby Warburg, pour être utilisée et consultées pendant ses études, conférences et publications ; avec, par la suite, la transformation de la bibliothèque en institut promoteur de la recherche, ouvert au public, elle a été repensée en tant que corpus et instrument. Mais selon un système, il est vrai, inédit et qui ne respecte pas les dispositions communes de classement pour la photographie de documentation historico-artistique - telles que le genre, la topographie, la chronologie, l'auteur.

11 La collection de photographies Warburg alimente aussi, matériellement le dialogue serré image/parole de la méthode d'étude de l'auteur. Et représente une forme et une épiphanie du projet Mnemosyne, compris comme nom tutélaire de l'entreprise culturelle warburgienne - titre de l'atlas et inscription située sur le seuil de la salle de lecture de la $\mathrm{KBW}^{10}$. Le plan inédit pour la Bildersammlung rentre exactement dans ce cadre. Bild, Wort und Bild, Orientierung und Bild, Handlung und Bild. Les quatre sections planifiées pour la Bildersammlung Warburg reflètent, en y répondant, l'organisation logique de la collection des livres de la bibliothèque, dédiée par Warburg à Mnemosyne, mère des muses. Elles indiquent, dans les branches des sous-catégories, la structure en efflorescence des documents sélectionnés et réorganisés pour le livre inachevé Mnemosyne. Et elles inventent un système, dans le sens d'organisation de la pensée, original en bibliothéconomie et en photothéconomie.

\section{Devise}

12 «"Das Wort zum Bild" ist die Devise unserer Bibliothek»: c'est ainsi que Warburg faisait référence à la devise (mais lisons aussi à la devise c'est-à-dire à la monnaie d'échange) de la KBW, à la fin d'une conférence en avril 1928 sur l'histoire des fêtes de cour.

13 Certains livres et images [terminait Warburg] qui ont été présentés ce soir, présents seulement dans des ouvrages très rares, sont ici disséminés dans la salle sur un des panneaux suspendus pour votre visite que vous ayez un aperçu de notre laboratorium ${ }^{11}$.

14 Les panneaux avec photographies et autres objets figurés constituaient un aspect de la pratique méthodologique Wort und Bild. On peut en effet observer que la limite entre biblio et iconothèque, dans certaines parties des collections respectives, s'efface conceptuellement. Cet effacement est mis en scène, physiquement, justement par la modalité de l'exposition. A la KBW, on a recours à l'exposition à l'occasion de conférences, visites, séminaires. Sur les panneaux, on accroche des photographies et souvent on y appose aussi des objets figurés comme des livres ou des documents illustrés imprimés, y compris des documents d'actualité, exposés en correspondance de la page avec la ou les figures concernées. Le panneau semble s'offrir comme une autre dimension de la Bibliothèque et de ses objectifs: une extension (le livre exposé) et une projection (l'image reproduite). Le projet et l'exposition des panneaux prennent la valeur d'une véritable projection, per exempla et à plusieurs niveaux, des documents des collections, des thématiques et des objets de la recherche promue par la bibliothèque, des moyens - méthodes et instruments - d'une telle recherche ${ }^{12}$.

15 Le binôme image/parole de cette devise exprime la relation entre tradition textuelle et iconographique, entre texte verbal et visuel, y compris dans le dialogue de la 
composition picturale avec les sources littéraires et philosophiques de référence. Dans ce cas d'étude particulier, les fêtes à la cour, le binôme s'incarne dans une catégorie précise de livres que Warburg définit «livres rares» (seltenen Bücher), comme les précieux livres illustrés de cérémonials ou Festival books, sans lesquels il serait impossible d'enregistrer le «missing link» - écrit-il dans ses notes - entre vie et $\operatorname{art}^{13}$.

La devise a aussi cet aspect pratique: image/parole est utilisé comme instrument de recherche, expérimenté par Warburg dans la structuration de ses conférences avec la lecture d'un texte d'introduction, la projection de diapositives, la visite guidée des panneaux réalisés chaque fois ad hoc en piochant dans le thesaurus de la collection photographique. C'est la méthode de travail par laquelle l'image est menée à la parole et conduite à communiquer, grâce au travail de l'auteur et à l'invention de dispositifs herméneutiques et des dispositifs d'exposition adaptés.

\section{La conférence sur les tapisseries Valois. Bildträger, iconophora}

La conférence sur les tapisseries Valois est le premier exemple d'exportation, depuis la Kulturwissenchaftliche Bibliothek Warburg de Hambourg, de cet aspect pratique de la méthode Wort und Bild.

18 A l'automne 1927 Warburg revient en Italie pour la première fois après le début de la première guerre mondiale. Le Kunsthistroriches Institut de Florence en 1927 réintègre le Palais Guadagni, après avoir été, provisoirement, pendant quatre ans, dans les murs des Offices $^{14}$. Le 29 octobre à la conférence d'inauguration Warburg parle d'une découverte qu'il avait faite environ 30 ans auparavant, concernant les fêtes médicéennes à la cour des Valois représentées sur une série de grandes tapisseries conservées au musée des Offices ${ }^{15}$.

Le Jahresbericht de l'Institut florentin rapporte aussi un commentaire technique sur l'événement, défini comme la présentation des résultats importants d'une recherche de vingt ans sur les tapisseries Valois: «sous forme de leçon avec un vaste matériel photographique» ${ }^{16}$. En effet, dans les (matériaux de travail qui attestent de l'événement, les références aux «images exposées» (ausgestellten Abbildungen) ${ }^{17}$ témoignent $\mathrm{du}$ projet d'organisation d'une petite exposition photographique. La structure des documents de travail pour cette conférence confirme le protocole adopté par Warburg au cours de ces années. Il n'existe pas de texte achevé mais un véritable canevas de mise en scène qui comprend tout ce qui est nécessaire à la performance publique: un texte introductif, des notes sur les sources, des documents et sujets spécifiques (dans ce cas, par exemple, la généalogie des Valois), des notes pour le commentaire des planches, auxquelles correspondent des schémas de montage, les lignes de conclusion, un recueil de notes aphoristiques rédigées avant et après la conférence.

20 En outre, plusieurs notes pour la conférence se rapportent directement aux panneaux (Tafeln) et donnent le plan et la liste des images exposées. Les notes que l'on retrouve dans le canevas (écrites par Bing avec des interventions de Warburg, datées du 22 octobre), comparées avec les plans de montage et la numérotation des images indiquent la disposition et séquence qui suivent. 
21 La première image était celle de l'aquarelle de Clouet avec les portraits Valois conservé aux Offices (Fig. 1).

\section{Fig. 1}

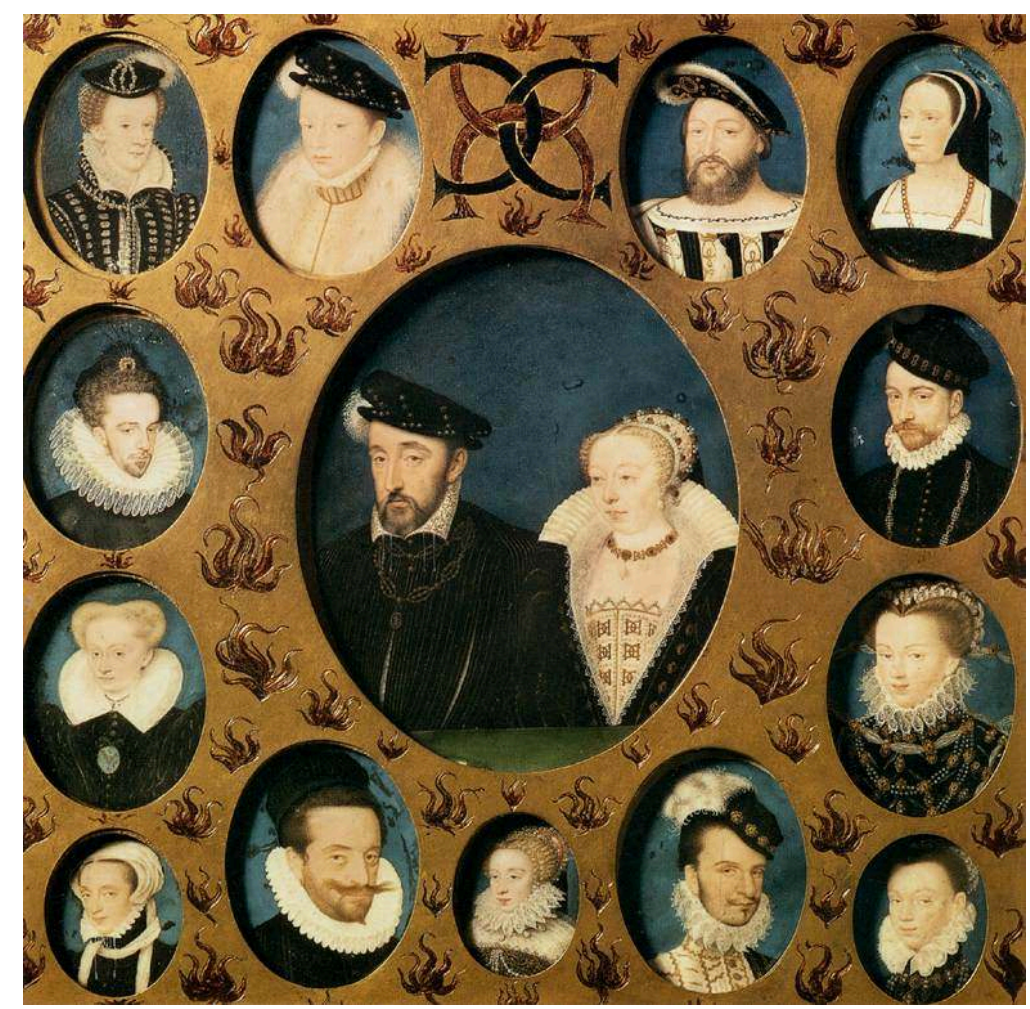

François Clouet (Tours 1515 ca.-Paris1572) et collaborateurs, Portrait d'Henri ll de Valois et Catherine de Médicis, après 1559, aquarelle sur parchemin, cm 11×9, Florence, Galerie des Offices, Couloir de Vasari, inv. 1890, n. 815

Florence elle même [écrit Warburg en introduisant cette image] possède dans un cadre collectif un recueil de merveilleux portraits de famille de l'arbre généalogique des Valois. Grâce à ce dernier, ainsi qu'à d'autres instruments iconographiques, il est possible d'identifier la plupart des personnes représentées sur les tapisseries ${ }^{18}$.

Les images suivantes étaient «8 photographies agrandies ${ }^{19} »-$ comme le précise Warburg dans son journal de bord - des tapisseries Valois, toujours aux Offices.

24 Après cette introduction, avec un tout premier plan sur les protagonistes de la discussion, les notes parlent de 14 planches, avec une numérotation progressive de chaque image.

25 - Sur la première planche, des sujets folkloriques (c'est l'exemple du Carême) avec l'art dévotionnel contemporain ${ }^{20}$;

- sur la deuxième planche, un exemple de sujets allégoriques faisant référence aux commandes des Médicis ${ }^{21}$;

- sur la troisième, des images de " divinités servantes » (servierende Gottes) d'après la description des fêtes pour les noces Sforza-Aragona de Pesaro, en comparaison avec des exemples d'autres thèmes d'antiquité (comme des sujets d'antiquité de Bellini, Squarcione, Mantegna) ${ }^{22}$;

- sur la quatrième planche, le sujet de la Punition d'Amour²3; 
29 comparaison avec la Punition d'Amour ${ }^{24}$;

30 Dürer ${ }^{25}$

31 - dans la septième planche, la figure de la Muse, dans sa forme antique, comme allégorie et en relation avec la musique (Pesaro, Mantegna, Filippino) ${ }^{26}$.

32 A propos du groupe de planches 4-7 et de leurs images nous lisons dans les notes de Warburg: «niveaux d'inflation du langage gestuel» (Inflationswerte der Gebärdensprache) ${ }^{27}$.

33 - Et encore, dans la huitième planche, Vices et Vertus, et leur lutte, en tant que sujet allégorique (Mantegna, Pollaiolo) ${ }^{28}$;

34 - la neuvième étape visuelle comprend un exemplaire du Balet comique de la royne de 1581 de Baldassarino, Baltasar de Beaujoyeul ${ }^{29}$.

35 Warburg encore à propos de ces images: « l'humanisation [mais nous pouvons lire aussi "incarnation"] des anciennes Pathosformeln / par le biais de / / l'Oeuvre récitative » (Die Humanisierung der antikischen Pathosformeln durch // die recitative Oper) ${ }^{30}$ :

36 - sur la planche dix, images d'après les dessins de Bernardo Buontalenti et autres pour Intermezzo de $1589^{31}$;

37 - sur la onzième planche, encore deux images d'appareils festifs, extraits de «livres rares» du XVIIe siècle ${ }^{32}$;

38 - sur la douzième planche, les images de deux entrées triomphales d'eau du XVII ${ }^{e}$, à Gand et Amsterdam et le char comme symbole de domination ${ }^{33}$;

39 - la séquence se termine (13, 14; Fig.2 $)^{34}$ avec une trouvaille warburgienne: avec les illustrations de Neptune du Virgile anglais de Dryden, un Neptune comme relief décoratif sur un arc factice pour le couronnement de Charles II, et encore Charles II luimême comme Neptune dans un sceau, une image moderne de domination et triomphe sur la mer: l'affiche de la coupe Schneider pour les courses d'hydravions ${ }^{35}$. 
Fig.2
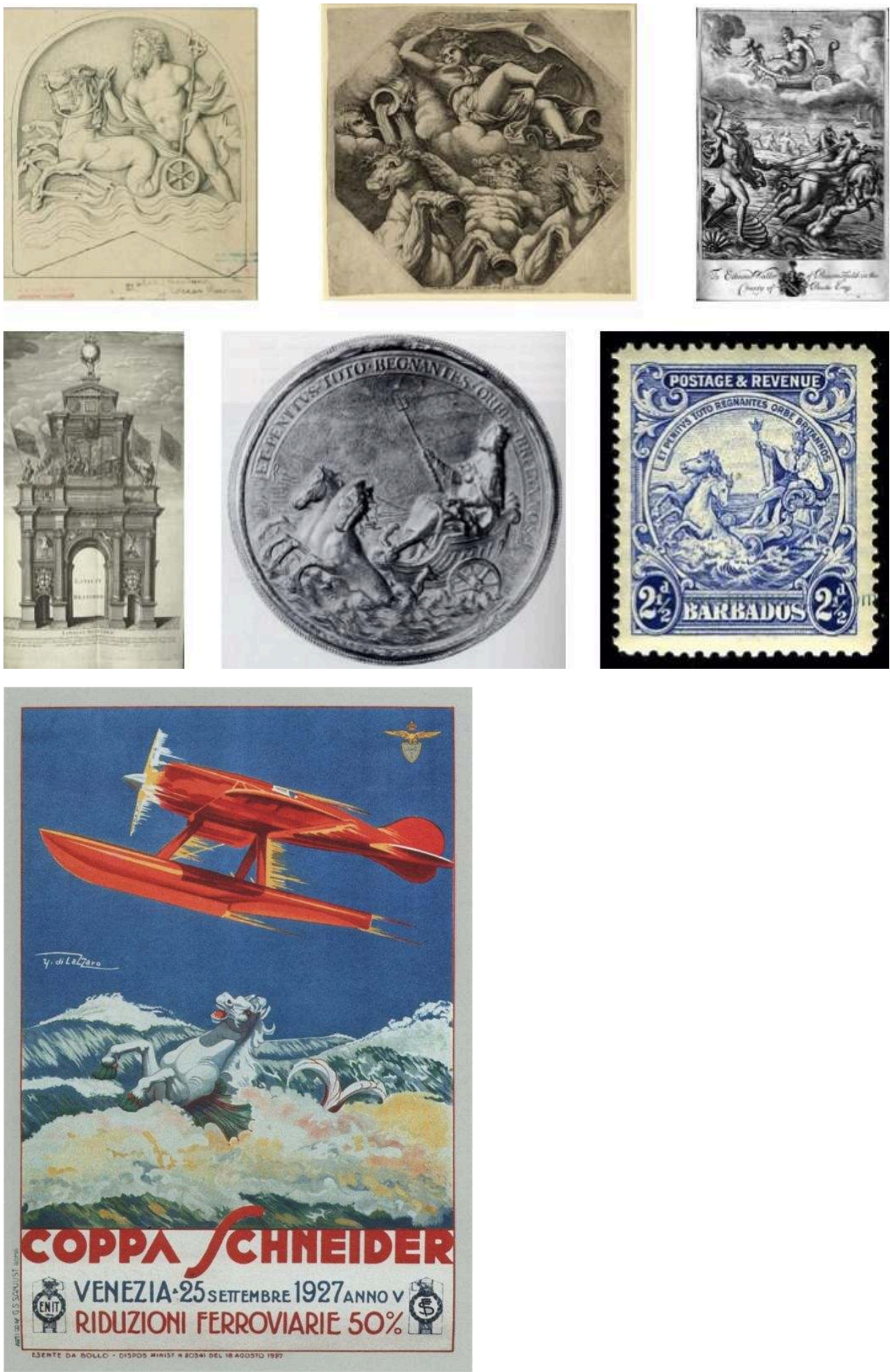

Images des planches 13 et 14

40 Comme dans le cas de la conférence à la Biblioteca Hertziana en 1929, il n'est aucune photographie qui témoigne de l'installation - alors que les panneaux préparés par Warburg à Hambourg, justement à partir de 1927 sont régulièrement et méthodiquement photographiés. Nous pouvons donc seulement avancer des 
hypothèses: une solution d'organisation semblable à celle qui fut adoptée dans la bibliothèque de Hambourg, ou alors, plus probablement, une sorte "d'installation pour le voyage», comme en témoignent les photographies des panneaux provisoires montés par Warburg dans son appartement au Palace Hôtel de Rome, utilisées pour préparer la conférence à la Biblioteca Hertziana, pour travailler sur Mnemosyne et pour présenter ses propres études au cours de conférences privées, toujours dans les chambres de l'Hôtel.

41 À la base de cette expérience, il est un module polyphonique pour la narration, ou, mieux, l'exposition historico-artistique qui se reflète et se concrétise dans l'utilisation systématique de la reproduction photographique, dans l'exploitation positive de certaines de ses potentialités, dans la mise au point d'une série d'expédients de composition graphique pour le montage.

Certains critères de composition, repérables seulement en partie sous forme d'instructions entre les notes de travail - par exemple certaines notes sur les passepartouts des photos ou dans les recueils aphoristiques de notes -, se répètent de manière presque inchangée depuis les expositions pour les conférences et, confrontés aux notes et notices correspondant aux matériaux objets reproduits et exposés, ils permettent l'identification d'expédients graphico-compositifs récurrents. Il est donc possible de mettre en évidence des moments de contextualisation (par l'insertion de détails multiples et de changement d'échelle), de comparaison en faisant ressortir les détails (par agrandissements, assemblages, répétitions), de mise en page critique (par construction de séquences signifiantes à l'intérieur de toute la mise en page). La multiplicité des critères et des modèles de photocomposition adoptés - versus une tendance évidente à l'uniformité, par ex. de sujet, de chronologie, de style dans les œuvres contemporaines - devait constituer la caractéristique particulière des planches du Bilderatlas, et du Mnemosyne en tant que livre extra-ordinaire. D'où une définition possible des montages warburgiens d'après le modèle polyphonique et le schéma théorique de la technique du contrepoint : chaque voix (de motif ou thème) jouit dans la composition d'un rôle qui lui appartient, indépendant, et mélodique et rythmique, évoluant avec les autres selon des principes harmoniques.

Dans le canevas de la conférence sur les tapisseries Valois, on peut lire:

Le devoir de mémoire/remémoration sociale en tant que fonction mnémique [nous lisons les lignes de conclusion du canevas pour la conférence] apparait ici claire comme le jour: par un contact toujours renouvelé avec les monuments du passé, elle préserve aussi la circulation de la lymphe à partir de la matrice du passé jusqu'à la forme antique, de sorte que la forme énergiquement chargée ne devienne pas un dynamogramme calligraphique ${ }^{36}$.

Comme on le sait, l'attention de Warburg est souvent attirée - c'est le cas ici - par des œuvres marquées de façon significative par un processus de transposition, reproduction ou qui présentent des caractères de reproductibilité et donc de transmission. La partie finale du texte de la conférence montre clairement que son attention se focalise sur les reconstructions de segments de succession et sur les supports, mécanismes et modes de transmission.

La mobilité spécifique des images pariétales tissées [nous continuons de lire dans le canevas de la conférence] représente le type le plus élevé de l'art de la reproduction à ses débuts, puisque l'original du temps - le carton, comme on l'appellera par la suite - 
peut servir comme modèle plusieurs fois. Il peut donc donner à la diffusion de son langage figuratif la même force que la mobilité de l'œuvre imprimée, qui, pour ce qui est aussi bien de l'image que de la parole imprimée, est un descendant démocratique en ligne directe des supports d'image tissés à la cour. Il est toutefois certain que la relation organique de la tapisserie, sorte de dispositif et de costume, avec son commanditaire à la cour est très différente de celle des œuvres imprimées avec leur acheteur ${ }^{37}$.

Dans une note pour la conférence, datée du 23 octobre, cette attention est formulée dans des termes synthétiques : "techno-dynamique des supports de l'image / La reproductibilité, la répétibilité / la mobilité des supports de l'image» ${ }^{38}$. Bildträgers: supports de l'image, véhicules, media - iconophora.

Dans ce sens, la conférence présente des sujets-clé pour les lineamenta de la théorie warburgienne sur la dynamique de la «soziale Mneme»: les fêtes à la cour comme des véhicules, éphémères, de la vie à l'art, des images (parfois immortalisées sur des tapisseries ou pérennisées sous la forme de "livre rare»); les tapisseries comme incunable, ou prototype de luxe, du medium reproductible (du carton/matrice) pour les images dans l'histoire. Le sujet de discussion permet de définir aussi un aspect pratique et méthodologique de la théorie: une partie du programme de la recherche se concentre sur la relation matérielle entre les épiphanies de figures de la culture visuelle d'un moment historique donné dans des contextes créatifs différents - depuis les plus éphémères comme le théâtre aux plus littéralement monumentaux comme la peinture - et la documentation correspondante - depuis les tapisseries, justement, jusqu'aux illustrations pour les livres, aux tableaux.

49 L'ikonologische Durcharbeitung que Warburg estime nécessaire pour la compréhension des «créatures préhistoriques»" telles que les tapisseries passe aussi par la reconnaissance de leur valeur documentaire et de Bildträger et de prototypes de la reproductibilité - sur un rayon contextuellement limité et déterminé.

\section{Leitmuschel}

Le 29 février 1928, Aby Warburg présente un discours conclusif au séminaire d'hiver de la Bibliothèque consacré à la méthode pour une «kunstwissenschaftliche kulturwissenchaft $»^{40}$. Les documents de travail sont constitués d'un texte introductif, des listes des images utilisées, des notes de montage et d'un texte de conclusion. Sur la feuille qui introduit les notes se rapportant de façon spécifique aux montages sur les panneaux préparés pour l'occasion, on peut lire:

51 En guise de conclusion ce soir, je veux expliquer encore une fois cette méthode au moyen de motifs pris, pour ainsi dire, comme moule-guide entre les couches des siècles: le Nachleben de l'arc de triomphe romain, de la forme gréco-mythique du héros mourant, des Muses et de Médée ${ }^{41}$.

52 Leitmuschel est le terme introduit au XIX ${ }^{\mathrm{e}}$ siècle en géologie pour définir la fonction géognostique des fossiles qui caractérisent de façon fiable la couche géologique à laquelle ils appartiennent. Le terme, qui s'est fixé ensuite dans la forme Leitfossil, est devenu un instrument d'indication et de différenciation des couches géologiques, permettant avec la biostratigraphie une émancipation de la discipline stratigraphique hors de l'intersection entre paléontologie et lithologie, lui permettant d'atteindre un status transdisciplinaire qui lui est propre ${ }^{42}$. Comme on peut le lire dans les notes de 
Warburg pour la conférence, la «Sirène», le "char tiré par des hippocampes» ${ }^{43}$ (le char de Neptune) sont, parmi les tapisseries Valois, des Leitmuscheln. A l'exemple de l'analyse biostratigraphique, on propose une morphologie iconologique construite, de façon aussi pratique, sur un réseau d'images.

Par rapport à l'adoption du système de montage de photographies à la Biblioteca Hertziana de Rome, pendant l'hiver 1928-29 lors du dernier voyage en Italie, on remarque un écart. ${ }^{44}$ Warburg propose de ne pas projeter de diapositives et d'exposer seulement les montages de photographies. Les jours suivant la conférence, il tient d'autres leçons avec le support des panneaux. Les matériaux, à nouveau préparés dans son appartement au Palace Hôtel de Rome, seront ensuite montrés à de petits groupes ou à des visiteurs isolés. Kenneth Clark mentionnera l'événement à la Hertziana comme étant un moment mémorable dans son parcours intellectuel ${ }^{45}$. Gombrich pointera l'excentricité d'un choix que le public a beaucoup de difficulté à voir et à apprécier ${ }^{46}$. «Ce sera», expliquait Warburg à Ludwig Curtius les jours précédant la conférence, «une sorte de démonstration anatomique sur la méthode de la science de l'art» ${ }^{47}$. On essaie de montrer la méthodologie même de la Kunstwissenschaft, au-delà de l'apparence et de la matière, ou mieux à travers celles-ci, de l'objet de recherche, qui est projeté et sublimé en image dans la reproduction photographique. Ce qui est exposé en tant que figure ou sous forme fossile, ce sont certains passages et organes du système de circulation de la lymphe vitale des images; fossile, donc, en tant qu'empreinte ou trace (d'un fragment) du visuel d'un moment historique culturel donné, qui identifié, analysé, exposé de façon appropriée, peut agir comme un indicateur.

\section{NOTES}

1. J'ai eu l'occasion d'analyser et reconstituer les documents d'origine de cette conférence en 2010 en tant que kurzeit Stipendiatin du Kunsthistoriches Institut à Florence.

2. Warburg Institute Archive (WIA), III.98.6.1, fol. 17 [6]; cf. WIA, General Correspondence (GC), 2 novembre 1927, Aby Warburg à Jacques Mesnil [Jean-Jacques Dwelshauvers]: «ikonologische Feststellung»; cf. III.98.3, «Eigenbericht an Bodmer durch G[ertrud]B[ing], 10 Nov. 927», «[...] benutzte neben gleichartigen Motiven auf den anderen Teppichen, deren Herkunft und Gestaltung ikonologisch verfolgt wurden». Le catalogue de la WIA est en cours de révision par Claudia Wedepohl, les références et signatures présentées ici doivent donc être considérées comme provisoires.

3. WIA III.98.6, fol. 20.

4. In Sitzungsberichte der Heidelberg Akademie der Wissenschaften, Philosophischistorische Klasse, Jahrgang 1920, n. 26, Heidelberg, 1920; La divination païnne et antique dans les écrits et les images à l'époque de Luther, in Aby Warburg, Essais florentins, Paris, Klincksieck, 1990, pp. 245-294.

5. Warburg, Heidnisch-antike Weissagung (1920), p.70.

6. Warburg, Heidnisch-antike Weissagung (1920): «Die Wiederbelebung der dämonischen Antike vollzieht sich dabei, wie wir sahen, durch ein Art polarer Funktion des ein fühlenden Bildgedächtnisses», p. 70.

7. Giorgio Agamben, Aby Warburg et la science sans nom [1975] in Image et mémoire, Paris, Hoëbeke, 1998, pp. 9-43.

8. Robert Klein, La forme et l'intelligible. Écrits sur la renaissance et l'art moderne, [1964] Gallimard, Paris 1970, p. 224

9. Katia Mazzucco, "L'iconoteca Warburg di Amburgo", in Rivista storica 3, décembre (2012).

10. Katia Mazzucco, "Mnemosyne, il nome della memoria. Bilderdemonstration, Bilderreihen, Bilderatlas: una cronologia documentaria del progetto warburghiano", in Quaderni del Centro Warburg Italia 4-6, 2006-2008 (2011), pp. 139-203 (cf. édition polonaise révisée “Mnemosyne. Chronologiczna prezentacja dokumentów związanych z Atlasem Warburg", in Konteksty, 2-3 (2011), pp. 120-142). 
11. WIA III.111 (1-2), Handelskammer, fol. 3 [25A]: «Einige Bücher und Bilder, die heute Abend vorgeführt wurden und nur in sehr seltenen Werken zu erfassen sind, sind hier im Saal auf einer von den Hängetafeln zu Ihrer Besichtigung ausgebreitet, um einen Einblick in unser Laboratorium zu geben».

12. “Mnemosyne. Chronologiczna prezentacja” (2011); Katia Mazzucco, "Quarant'anni di bibliofilia e iconofilia. Osservazioni sul montaggio del libro Mnemosyne di Aby Warburg", in Rivista di Storia della Filosofia, 2 (2011), pp. 303-338. Une sélection de documents sur Ausstellungen et Bilderreihen organisée à la Kulturwissenschaftliche Bibliothek Warburg est maintenant publiée dans le Gesammelte Schriften - Studienausgabe de Warburg, Bilderreihen und Ausstellungen, U. Fleckner, I. Woldt (Hg.), Berlin 2012.

13. WIA III., fol. 83 [3]: «Das italienische Festwesen in seiner höheren Form ist ein wahrer Uebergang aus dem Leben in die Kunst. / C. d. R. (1860), s. 401»: ic Warburg cite la Kultur der Renaissance in Italien de Burckhardt dans le texte de la conférence à Florence en 1927.

14. Hans W. Hubert, L'Istituto germanico di storia dell'arte di Firenze, il Ventilabro, Firenze 1997.

15. Les remarques formulées par la suite sont tirées d'une édition des documents visuels et textuels de cette conférence, en cours de publications sous ma direction

16. "Kunsthistorisches Institut in Florenz. Jahresbericht" 1927/28, ss. 3.

17. WIA III.108.12, fol. 14

18. WIA III.98.6, fol. 20: «Familienportraits der Valois / Florenz selbst besitzt in einem Sammelrahmen eine Sammlung von Köstlichen Familienportraits zum Stammbaume der Valois. Durch dies u.[nd] andere ikonographische Hilfsmittel lässt sich die bei weitem grösste Anzahl der auf den Teppichen dargestellten Personen identifizieren».

19. WIA III.16, Florence, 1927, fol. 6.

20. WIA III.108.12, fol. 21.

21. WIA III.98.6.1, fol. 67; WIA III.108.12, fol. 22.

22. WIA III.98.6.1, fol. 69; WIA III.108.12, fol. 23

23. WIA III.108.12, fol. 24

24. WIA III.108.12, fol. 25

25. WIA III.108.12, fol. 26

26. WIA III.98.6.1, fol. 71; WIA III.108.12, fol. 27

27. WIA III.98.6.1, fol. 70

28. WIA III.98.6.1, fol. 72; WIA III.108.12, fol. 28

29. WIA III.98.6.1, fol. 74; WIA III.108.12, fol. 29. Selon toute probabilità, Warburg tenait en main l'exemplaire qu'il avait emprunté à la Bibliothèque du Istituto Musicale de Florence, précisément entre le 28 et le 31 octobre 1927 [cf. WIA ZK 5 Festwesen Frankreicht, fiche de prêt de la bibliothèque] et/ou celui-ci circulait dans l'assistance, et/ou, encore, était en libre consultation durant la conférence.

30. WIA III.98.6.1, fol. 73

31. WIA III.98.6.1, fol. 74; WIA III.108.12, fol. 30.

32. WIA III.98.6.1, fol. 76; WIA III.108.12, fol. 31.

33. WIA III.108.12, fol. 32

34. Images des planches 13 et 14: dessin d'un relief antique avec Neptune, par Johannes Adolph Overbeck, Atlas des griechischen Kunstmythologie, Bd. 3, Leipzig 1895, Taf. XII, Abb. 21; Pietro del Po (par Giulio Romano), Psyché enlevée par Zéfir et Neptune sur son char, gravure sur cuivre, 1650-1670; W. Hollar, F. Cleyn, Neptun, da The works of Virgil containing his Pastorals Georgics, and Aeneis, translated into English verse by Mr. Dryden, adorn'd with a hundred sculptures, 2nd ed. London, Printed for Jacob Tonson, at the Judges-Head in Fleetstreet, near the Inner-Temple-Gate, 1698; David Loggan, Neptuno britannico, arc de triomphe pour l'entrée de Charles II à Londres le 23 avril 1661 par John Ogilby, The Relation of his Majesty's entertainment passing thorough the City of London to his coronation: Containing an exact Accompt of the whole Solemnity the Triumphal Arches, and Cavalcade, delineated in Sculpture; the Speeches and Impresses illustrated from Antiquity. To these is added A brief Narrative of His Majestie's Solemn Coronation: with His Magnificent Proceding, and Royal Fest in Westminster-Hall. By John Ogilby, London, printed by Thomas Roycroft, and are to be had the Authors House in Kings Head Court within Shoe-Lane, MDCLXII (with plates by David Loggan) 1661; George Vertue (da Thomas Simon), Charles II avec Neptune sur le char tiré par des hippocampes, sceau (1662) avec la devise ET PETIVS TOTO REGNANTES ORBI BRITANNOS, gravure, tiré de Medals, Coins, Great Seals and other Works of Thomas Simon, London 1753, Pl. XXXIII; Le Roi d'Angleterre avec Neptune, timbre de la Barbade, 1925 (?), 2 1² p, devise ET PENITVS TOTO REGNANTES ORBE BRITANNOS. 35. WIA III.98.6.1, fol. 77-78; WIA III.108.12, fol. 32-33. 
36. WIA III.98.6, fol. 82 [2] «Die Aufgabe des sozialen Gedächtnisses tritt hierbei als mnemische Funktion klar zu Tage<:> durch stets erneute Berührung mit den Denkmälern der Vergangenheit selbst das Säftesteigen aus dem Muttergrund der Vergangenheit bis in die antikisierende Gestaltung zu wahren, damit nicht aus der energetisch-erfüllten Gestaltung ein kalligraphiertes Dynamogramm wird».

37. WIA III.98.6, fol. 13 [2]: «[...] die spezifische Beweglichkeit der gewirkten Bilderwand einerseits <* gleich zu <in ihrem> Anfang*> den höchsten Typus der reproduzierenden Künsten darstellt, insofern die zeitgenössische Vorlage - der Karton, wie man später sagte - beliebig oft als Vorlage <solche> dienen kann u.[nd] somit der Verbreitung seiner Bildersprache denselben Nachdruck der $W<w>$ andernden Beweg<ch>lichkeit verleihen kann, wie das Druckwerk, das sowol was den Bilddruck wie den Wortdruck angeht, eigentlich in direkter Linie ein demokratischer Abkömmling des gewirkten höfischen Bildträgers ist. Allerdings kommt bei dem Bildteppich abweichend hinzu, dass er mit seinem höfischen Besteller gleichsam G<g>erät-u.trachtenmässig in ganz andrer organischer Weise verbunden ist, als etwa der Käufer mit Druckwerk».

38. WIA III.98.6.1, fol. 87: «Technisch-Dynamisches d. Bildträgers / die Reproduzierbarkeit / die Wiederholungsmöglichkeit / die Beweglichkeit d. Bildträgers».

39. WIA III.98.6.1, fol. 12.

40. WIA III.113.5.1, (Burckhardt-Übungen), illustrations, fol.6.

41. WIA III.113.4 (Burckhardt-Übungen), Schlussübung, fol. 20: «Diese Methode will ich nun / heute Ab[en]d. z.[u] Schluss noch einmal / durch die Schichten des Jahrhunderts gleichsam als Leitmuschel / durch [?] Motive aufzeigen / An dem Nachleben / des römischen Triumphbogens / an der griechisch mytischen / Gestaltung des sterbenden Heroen / Der Musen / und der Medea».

42. Sur la Leitfossil et Warburg, voir Georges Didi-Huberman, L'Image survivante Histoire de l'art et temps des fantômes selon Aby Warburg, Les édition de Minuit, Paris 2002; cf. Claudia Wedepohl, "Ideengeographie". Ein Versuch zu Aby Warburg's "Wanderstrassen der Kultur", in Helga Mitterbauer, Katharina Scherke (Hg), Entgrenzte Räume. Kulturelle Transfers um 1900 und in der Gegenwart, Passagen, Wien 2005, pp. 227-254.

43. WIA III.98.6.1, fol. 112.

44. 19 gennaio 1929, Die römische Antike in der Werkstatt Ghirlandajo's, WIA III.115 (Hertziana).

45. The lecture which changed my life, in Kenneth Clark, Another Part of the Wood: a Selfportrait, Murrey, London 1974.

46. Ernst H. Gombrich, Aby Warburg: an Intellectual Biography, The Warburg Institute, University of London, London 1970.

47. WIA GC, 13 décembre 1928, Aby Warburg à Ludwig Curtius.

\section{INDEX}

Mots-clés : Interprétation, iconologique, figures, thèmes, image, parole, mémoire visuelle, empathie 


\section{AUTEURS}

\section{KATIA MAZZUCCO}

Docteur en histoire de l'art (Université de Sienne, 2006), spécialiste de l'héritage de la tradition classique dans l'art et la culture visuelle contemporaines; elle a été assistante d'enseignement pour le cours d'Iconologie, Histoire de la tradition classique, Histoire de l'art antique et l'archéologie de l'Université Ca 'Foscari et IUAV de Venise. Elle a été bénéficiaire d'une bourse de recherche post doctorale à l'IUAV de Venise (2008), chargée de cours à la photothèque de l'Institut Kunsthistorisches à Florence (2010), Grete Sondheimer Fellow à l'Institut Warburg à Londres (2010), British Academy Visiting Scholar à Londres (2011). S'occupant depuis des années de Warburg Kreis et d'historiographie visuelle, elle est actuellement engagée dans un projet sur la photographie comme méthode de recherche pour l'histoire de l'art. Parmi ses publications: (avec K. W. Forster), Introduzione ad Aby Warburg e all'Atlante della Memoria, (Milano 2002); Prefazione a E. H. Gombrich, Aby Warburg. Una biografia intellettuale (Milano 2003); “Quarant'anni di bibliofilia e iconofilia. Osservazioni sul montaggio del libro Mnemosyne di Aby Warburg", Rivista di Storia della Filosofia, 2 (2011); “1941, English Art and the Mediterranean. A Photographic Exhibition by the Warburg Institute in London", in Journal of Art Historiography, 5 (2011) 